\title{
Renal lymphatics, and lymphatic involvement in sinus vein invasive (pT3b) clear cell renal cell carcinoma: a study of $\mathbf{4 0}$ cases
}

\author{
Stephen M Bonsib \\ Department of Pathology and Laboratory Medicine, Indiana University School of Medicine, University \\ Hospital, Indianapolis, IN, USA
}

\begin{abstract}
Although renal sinus vein invasion is the most common site of extrarenal involvement in clear cell renal cell carcinoma (CC), CC also spreads by lymphatics. As cortical lymphatics drain into the sinus, some involved sinus structures may be lymphatics, not veins. This possibility was investigated with podoplanin, a specific lymphatic endothelial marker, in $40 \mathrm{CC}$ with sinus vein invasion. Ten blocks of uninvolved kidney, serving as controls, showed lymphatics within the adventitia of midcortical intralobular arteries. Lymphatics became more numerous and enlarged with progression towards the medulla. No lymphatics were among glomeruli or within the medulla unless associated with inflammation. The largest lymphatics occurred within the sinus, and were also noted within pelvic muscularis, and media of large veins. Intralymphatic tumor was observed and divided into two Groups. Group 1 (four cases) involved lymphatics within the invasive edge of tumors lacking a pseudocapsule. The lymphatics were small $(0.045-0.19 \mathrm{~mm})$, irregularly shaped, often incomplete, and contained single cells or small clusters of tumor cells. Group 2 (four cases) involved sinus lymphatics separate from tumor. One case each also involved adventitial lymphatics of an intralobular artery, the muscularis of the renal pelvis, and media of a muscular vein. The intralymphatic tumor in Group 2 often appeared discohesive, not endothelial cell invested, and larger than in Group 1 (0.4-0.5 mm). Conversely, tumor within muscular veins was cohesive, contained a capillary plexis, and was endothelial cell invested. In conclusion, intralymphatic tumor can be demonstrated in CC. Lymphatic involvement is less frequent than venous involvement and involves smaller structures. The potential for lymphatic spread may not be equal among involved lymphatics. Small peritumoral lymphatics may be destined for destruction by tumor growth. However, involved lymphatics within sinus and associated with renal pelvis, are likely sources for lymphatic spread and lymph node metastases.

Modern Pathology (2006) 19, 746-753. doi:10.1038/modpathol.3800589; published online 17 March 2006
\end{abstract}

Keywords: renal cell carcinoma; clear cell renal cell carcinoma; lymphatic invasion; lymphatic endothelium; podoplanin

Renal cell carcinoma preferentially extends beyond the kidney by invasion of the renal sinus. ${ }^{1,2}$ This is usually associated with invasion of renal sinus veins and is likely responsible for subsequent development of hematogenous metastases to lung, liver, bone, and other sites. Lymphatic invasion also occurs in some renal cell carcinomas since lymph node metastases develop. Lymph node dissections have demonstrated that from 7 to $17 \%$ of patients

Correspondence: Dr SM Bonsib, MD, Department of Pathology and Laboratory Medicine, Indiana University School of Medicine, University Hospital, UH 3465, 550 North University Blvd, Indianapolis, IN 46202, USA.

E-mail: sbonsib@iupui.edu

Received 20 December 2005; revised 22 February 2006; accepted 23 February 2006; published online 17 March 2006 have hilar or locoregional lymph node metastases. ${ }^{3,4}$ Distinguishing venous involvement from lymphatic involvement in human cancers has been hampered by the difficulty in distinguishing veins from lymphatics. Although lymphatics show distinctive ultrastructural differences, such as gaps between endothelial cells, and absent to interrupted basal lamina, smooth muscle and pericytic investments, histologic appreciation of these ultrastructural features is not feasible. ${ }^{5-7}$ In the past several years a number of specific lymphatic endothelial cells markers have become available, such as podoplanin, lymphatic vessel endothelial hyaluronan receptor-1, vascular endothelium growth factor receptor-3, D240, and Prox-1. ${ }^{8-11}$ This has enabled investigators to investigate the incidence and prognostic importance of lymphatic invasion in a number of cancers, and 
has led to the identification of tumor-associated lymphangiogenesis. ${ }^{12-16}$ Renal cell carcinoma has not been previously examined for lymphatic invasion using lymphatic-specific markers. In this study, 40 cases of sinus vein invasive clear cell renal cell carcinoma (CC) have been examined for lymphatic involvement.

\section{Materials and methods}

In this study, 40 cases of renal sinus vein invasive (pT3b) clear cell renal cell carcinomas were studied. Two blocks from each case were selected that demonstrated invasion of muscular renal sinus veins and contained a portion of the primary tumor. Immunoperoxidase stains were performed using podoplanin (AngioBio Co, Del Mar, CA, USA, 1:100), and a cocktail containing both CD 31 and smooth muscle actin (Abcam, 1:1000). Ten controls consisted of one block of uninvolved tissue completely separate from tumor that contained both cortex and medulla. This was employed to delineate the appearance and distribution of lymphatics in non-neoplastic areas of the kidney. The diameter of lymphatics involved by tumor was measured using an ocular micrometer. The structure measured was required to have a nearly circular profile, with the shortest axis used to define the internal or luminal diameter. A lymphatic is defined as a podoplaninpositive endothelial lined structure. Intralymphatic tumor is defined by the presence of tumor in a space, partially or completed encircled by a podoplanin positive endothelial cell layer.

\section{Results}

Normal Lymphatics of the Cortex, Medulla, and Renal Sinus

The cortex and medulla of six of the 10 control kidneys appeared essentially normal aside from occasional subcapsular sclerotic nephrons with minimal-associated inflammation. Four control kidneys contained focal variably sized zones of prominent inflammation in the cortex and the medulla. Lymphatics, defined as podoplanin-positive endothelial lined structures, were identified in all 10 control kidneys, and easily distinguished from hematogenous capillaries, arterioles, arteries, and veins, which had podoplanin negative endothelial cell linings (Figure 1). In the histologically normal control kidneys, no lymphatics were observed in the superficial cortex. In the midcortex, one or more small lymphatics, the caliber of peritubular capillaries, were identified within connective tissue adjacent to intralobular arteries (Figure 1). No lymphatics were noted among glomeruli and tubules.

With progression from the midcortex to the corticomedullary junction, lymphatics became more

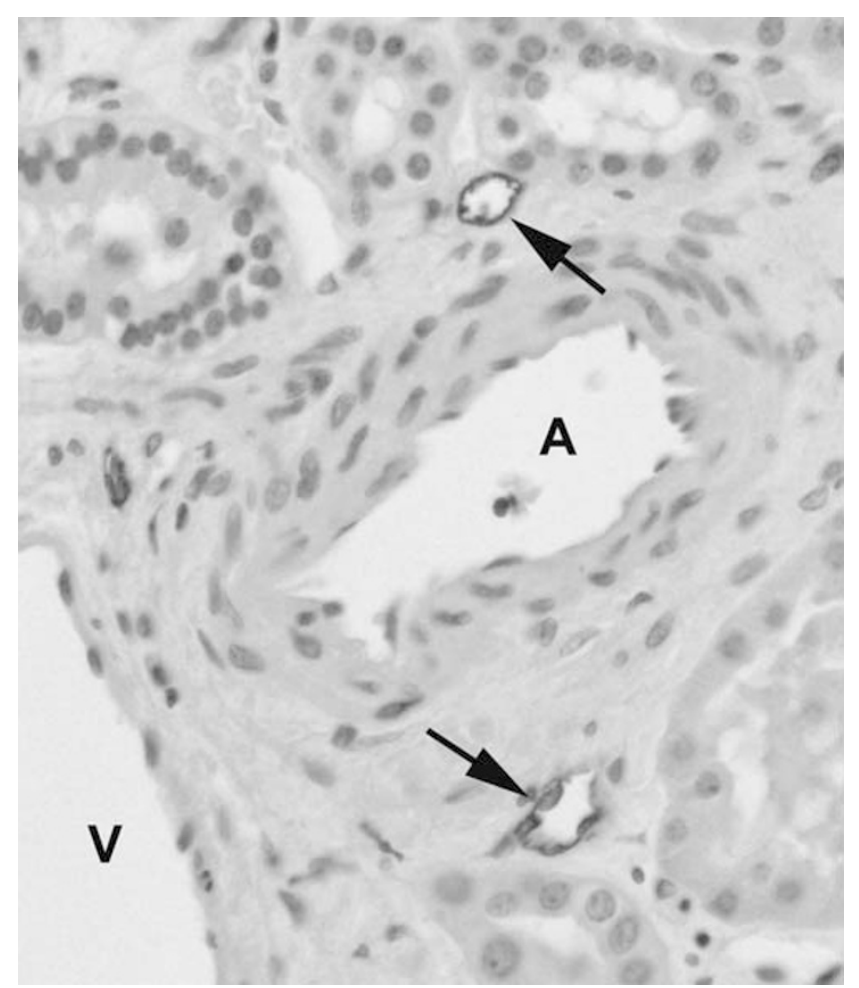

Figure 1 Midcortex of control kidney. The endothelium of an intralobular artery (A) and vein (V) are podoplanin negative, while the lymphatics in the periarterial adventitia (arrows) are positive for podoplanin. Podoplanin immunoperoxidase stain.

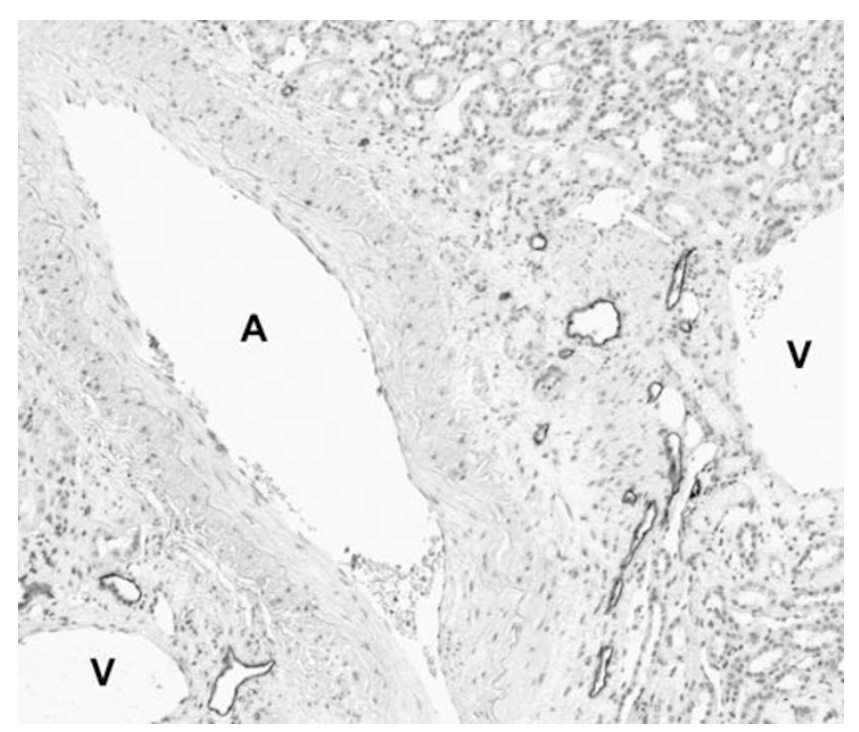

Figure 2 Corticomedullary junction of control kidney. Podoplanin-stained lymphatics are numerous, larger than those of the midcortex, and no longer limited to the periarterial adventitia. A, artery; V, vein. Podoplanin immunoperoxidase stain.

numerous and enlarged. At the corticomedullary junction lymphatics strayed from an arterial adventitial location noted within the cortex, but remained in the arterial venous structure (Figure 2). No lymphatics were observed within the inner or outer 

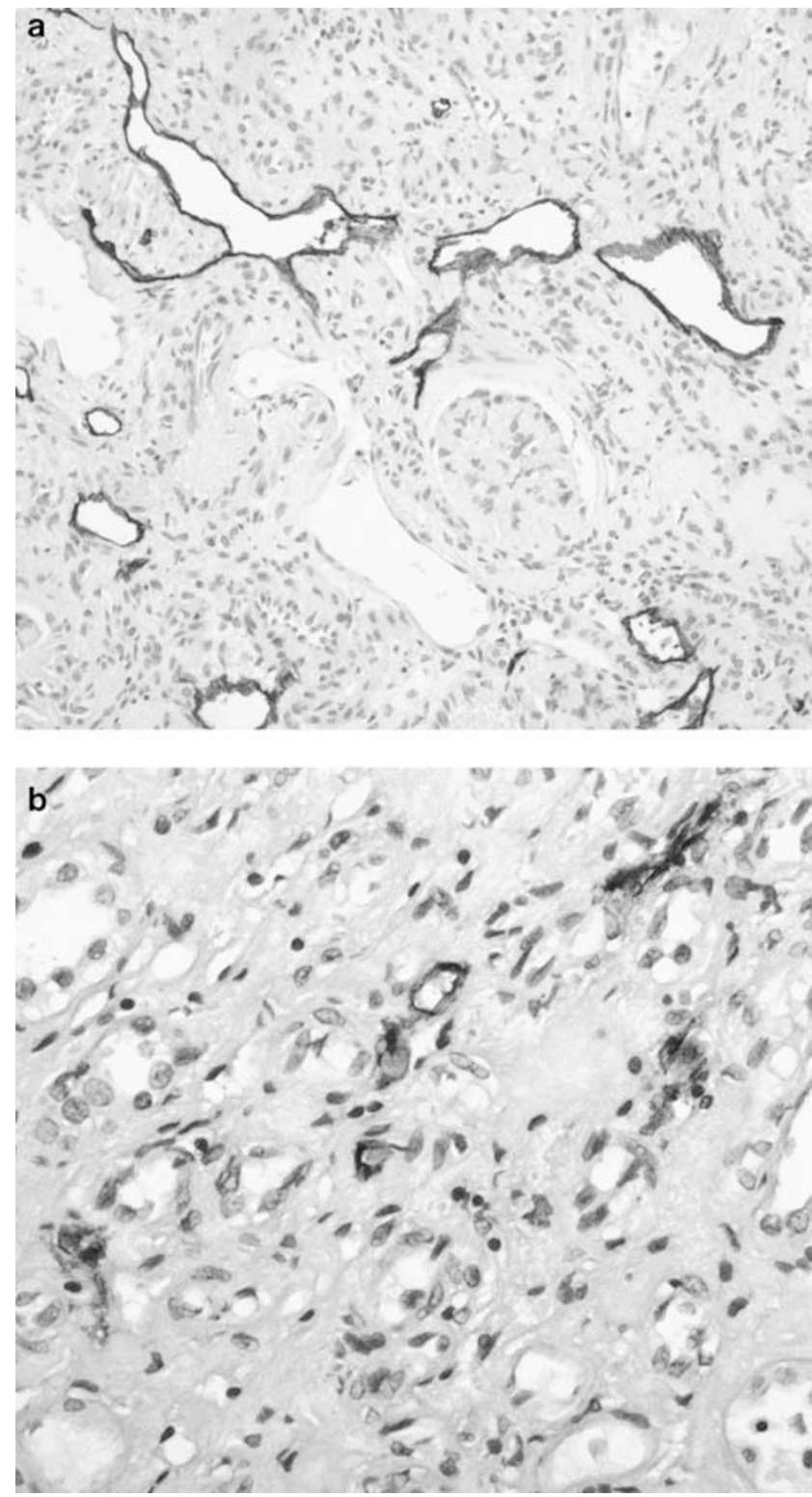

Figure 3 (a) Inflamed control kidney. Numerous podoplaninstained lymphatics are present amidst glomeruli and tubules within inflamed cortex. Podoplanin immunoperoxidase stain. (b) Medulla of control kidney. Several podoplanin-stained lymphatics are present within the vasa recta of the mildly inflamed outer medulla. Podoplanin immunoperoxidase stain.

medulla. Although the lymphatics in all levels of the cortex were smaller than the adjacent veins and lacked a discernable media, there was no predictable location or distinguishing feature on corresponding H\&E stained sections that would permit identification of a lymphovascular structure as a lymphatic, vs a vein or a hematogenous capillary, without a lymphatic endothelium stain.

In the inflamed control kidneys there was a close association between prominent zones of inflammation and lymphatics. The lymphatics were at times numerous and in locations not normally endowed

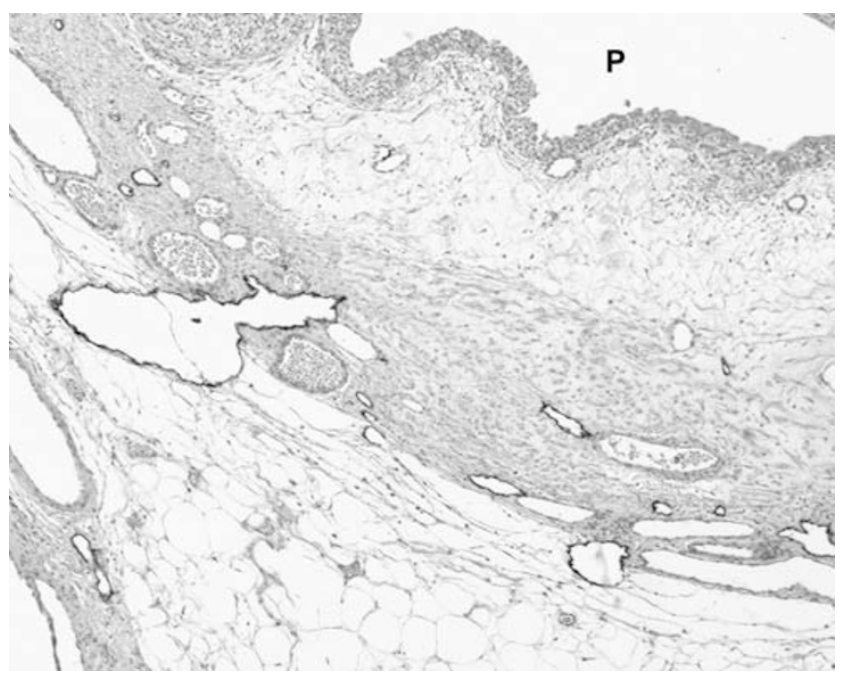

Figure 4 Control kidney. The muscularis of the renal pelvis (P) demonstrates a rich lymphatic investment. Podoplanin immunoperoxidase stain.

with lymphatics, located amidst cortical glomeruli and tubules (Figure 3a). Sometimes the lymphatics appeared less well formed, lacking a patent lumen, or appeared as a thin single-cell layer. Occasional lymphatics were also observed within inflamed medulla. In the outer medulla one or several small capillary sized lymphatics were observed associated with the vasa recta vascular bundles (Figure 3b). A rare individual lymphatic was noted within the inner medulla.

The largest caliber lymphatics were observed within the renal sinus. Within the renal sinus lymphatics lost an arterial association and were observed scattered throughout the sinus fat. There were numerous lymphatics within the renal pelvic muscularis (Figure 4). Lymphatics were also observed within the media of muscular sinus veins (Figure 5). Rarely lymphatics within the sinus fat had an interrupted smooth muscle media, but most consisted of a thin endothelial cell layer as the lymphatics within the renal parenchyma (Figure 6).

\section{Renal Lymphatic Invasion}

Two blocks of tumor from 40 cases showing renal sinus vein involvement and containing a portion of the primary tumor were selected for podoplanin staining. No lymphatics were identified within the substance of the primary tumor. At the periphery of the tumor, however, clusters of lymphatics were noted in 12 of 40 cases. In seven of 12 cases the lymphatics were outside the pseudocapsule, associated with a prominent inflammatory response. These peritumoral lymphatics were numerous small capillary sized structures, or slightly larger, sometimes located within glomeruli and tubules. Some peritumoral lymphatics possessed a lumen, but on occasion they appeared to consist as an elongated single layer of endothelium without a lumen. Five of 


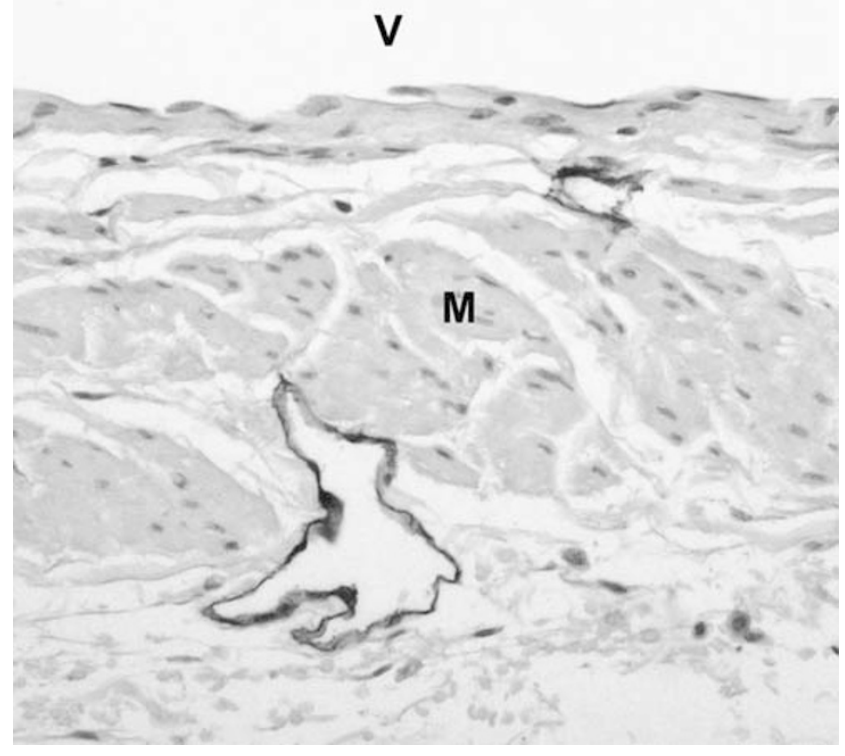

Figure 5 Control kidney. Lymphatics were observed within the media of a muscular vein (V). $\mathrm{M}$, smooth muscle media. Podoplanin immunoperoxidase stain.

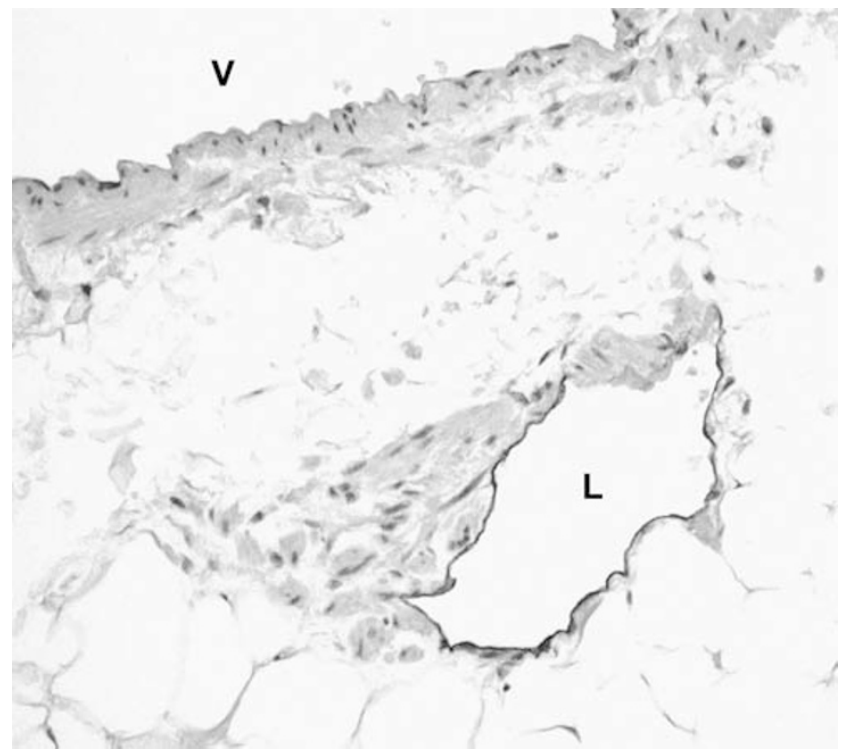

Figure 6 Renal sinus of control kidney. Some lymphatics within the sinus are larger than those in the renal parenchyma. This lymphatic has an interrupted a smooth muscle media. V, vein; L, lymphatic. Podoplanin immunoperoxidase stain.

12 cases showed an intermingling of small lymphatics with tumor cell at an invasive edge. Four of these contained intralymphatic tumor (Group 1) as discussed below.

Intralymphatic tumor was observed in seven of 40 cases. The cases were divided into two groups distinguished by whether the involved lymphatics were peritumoral, that is, immediately adjacent to the tumor within its invasive edge (Group 1), vs embolic, that is, completely separate from the

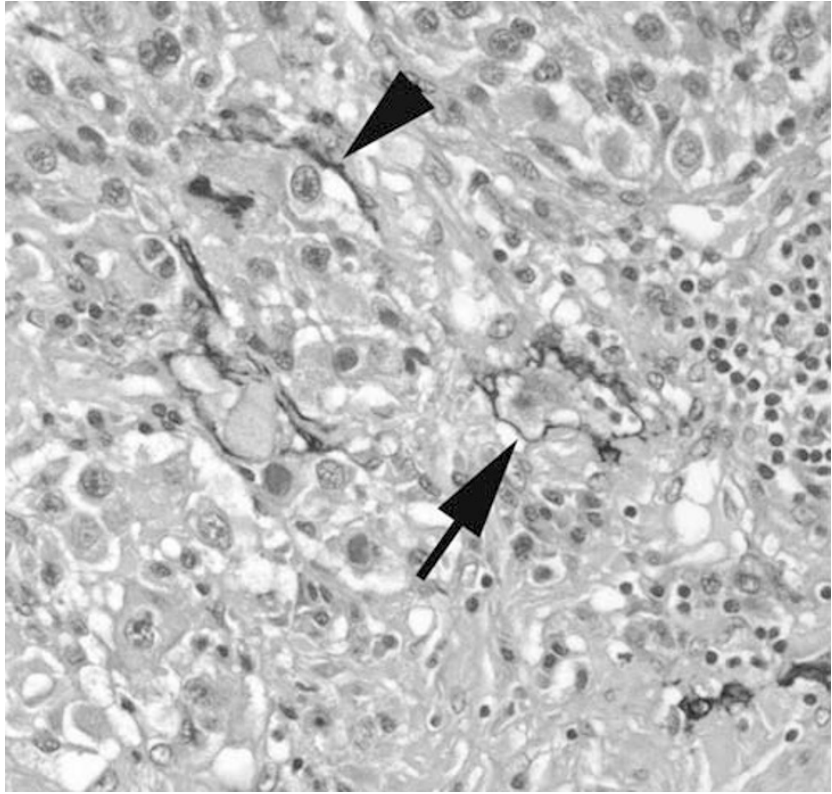

Figure 7 The invasive front of this tumor contains intralymphatic tumor (arrow). Notice the single attenuated cell of a possibly partially ablated lymphatic (arrowhead). Podoplanin immunoperoxidase stain.

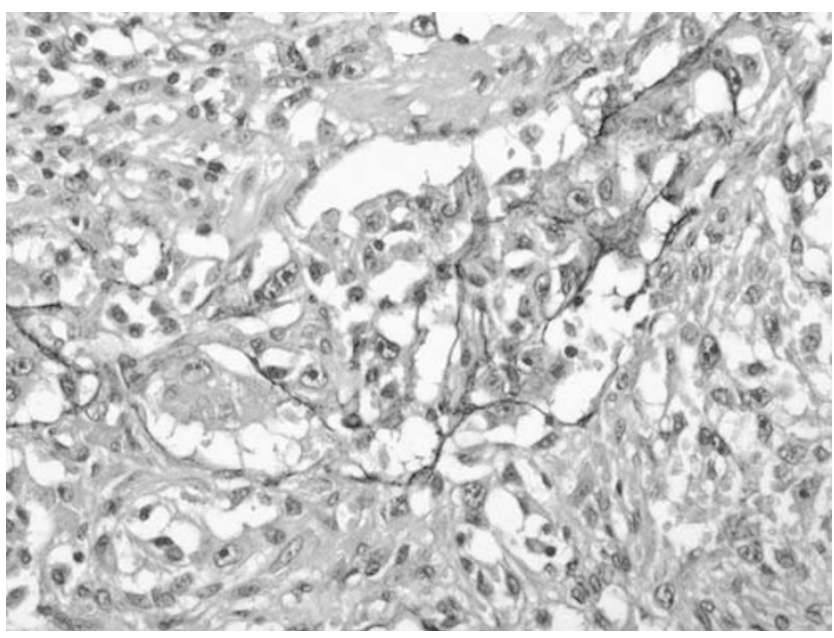

Figure 8 This is a tangled mixture of lymphatic endothelium and tumor at the invasive front of the tumor. Podoplanin immunoperoxidase stain.

primary tumor (Group 2). One case involved lymphatics of both types and is included in both Groups 1 and 2. There were four cases in Group1. At an invasive edge of the primary tumor where a pseudocapsule was absent, clusters of small lymphatics were present; a portion of these lymphatics contained individual tumor cells or small clusters of tumor cells (Figure 7). The involved lymphatics were small, often irregularly shaped, 0.045-0.19 mm in luminal diameter. Some lymphatics containing tumor appeared incomplete, or were a disorganized admixture of podoplanin-stained cells and tumor cells (Figure 8). 


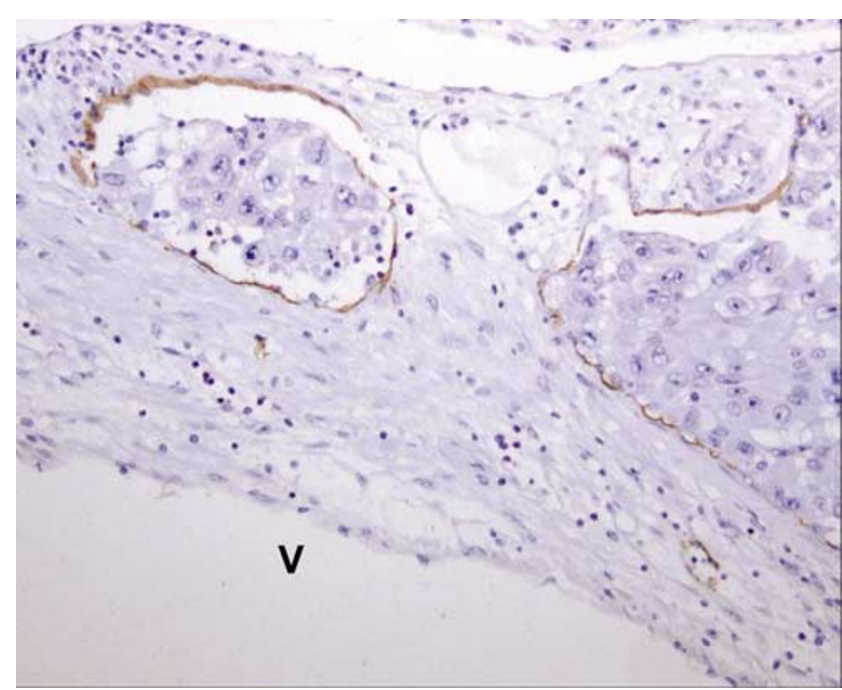

Figure 9 These sinus lymphatics completely separate for the primary tumor, are involved by tumor. V, vein. Podoplanin immunoperoxidase stain.

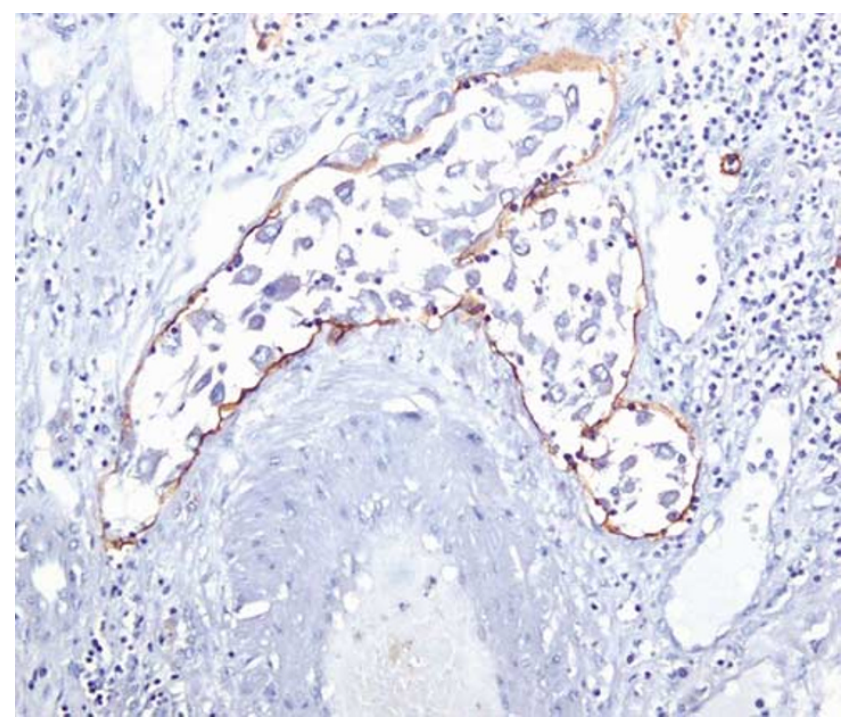

Figure 10 The adventitial lymphatics of this intralobular artery distant from the primary tumor are distended by tumor cells. Podoplanin immunoperoxidase stain.

There were four cases in Group 2. Intralymphatic tumor was observed within the renal sinus fat completely separate from the primary tumor in all four cases (Figure 9). One case also involved adventitial lymphatics of a cortical intralobular artery several millimeters from the primary tumor (Figure 10). One case also involved lymphatics within the muscularis of the renal pelvis (Figure 11). One case also involved lymphatics within the media of a large muscular vein (Figure 12a, b). The intralymphatic tumor cells often appeared discohesive, were not endothelial cell invested, and slightly larger than those of Group 1, measuring up to 0.4$0.5 \mathrm{~mm}$ in diameter in all four cases. However, this

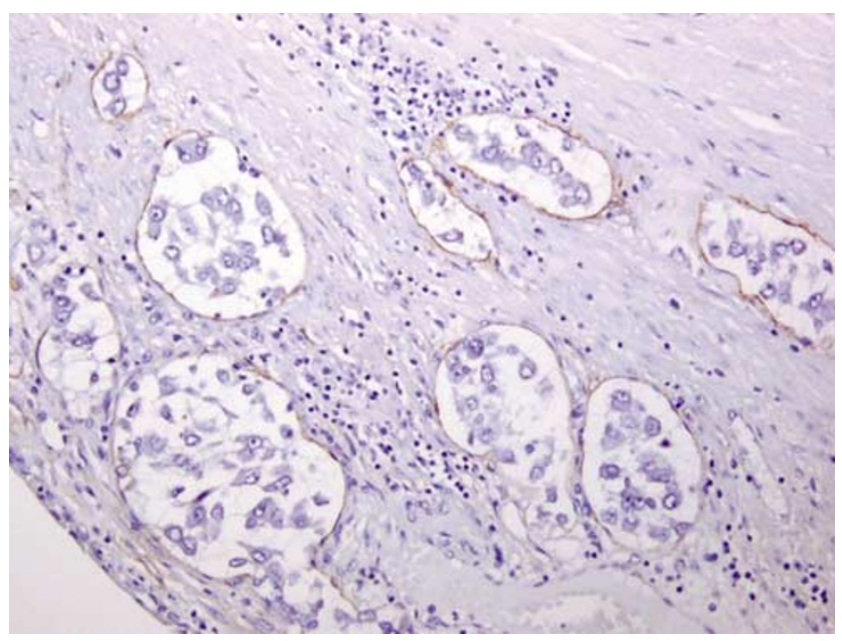

Figure 11 Wall of the renal pelvis showing lamina propria and muscularis lymphatics distended by tumor. Podoplanin immunoperoxidase stain.

was a fraction the size of the involved muscular veins that ranged from 1 to several millimeters in luminal diameter. No involved lymphatics demonstrated medial smooth muscle. Conversely, tumor within adjacent large to medium-sized muscular veins was cohesive, contained an intravenous capillary plexis (Figure 13), and usually endothelial cell invested at its periphery.

Although the number of cases with intralymphatic tumor is small, there was a correlation between intralymphatic tumor emboli and hilar lymph node involvement. Of the four cases in Group 2 containing lymphatic tumor emboli, three cases had lymph nodes examined. Twenty of 42 hilar and regional lymph nodes were involved in two of these three cases, 17 of 29 lymph nodes and three of 13 lymph nodes, respectively. Conversely, hilar lymphatics were negative in the three cases of Group 1 with intralymphatic tumor limited to peritumoral lymphatics (total of 39 lymph nodes examined). However, single hilar lymph node metastasis was also present in five of 33 cases in which intralymphatic tumor was not identified (total of 108 lymph nodes examined).

Follow-up data were available for 32 of 40 cases (Table 1). Of 32 cases, 18 (56\%) developed metastases (12 cases) or died of disease (six cases). Of the 12 patients alive with metastases, the follow-up period was short in 11, less than 2 years. Although 14 patients are currently alive and well, their average follow-up period is also short, only 14 months. As nine of these have had less than 1 year follow-up, their prognosis remains guarded.

\section{Discussion}

Renal sinus invasion represents the most common site of extrarenal extension in CC and has been shown to convey adverse prognostic impor- 

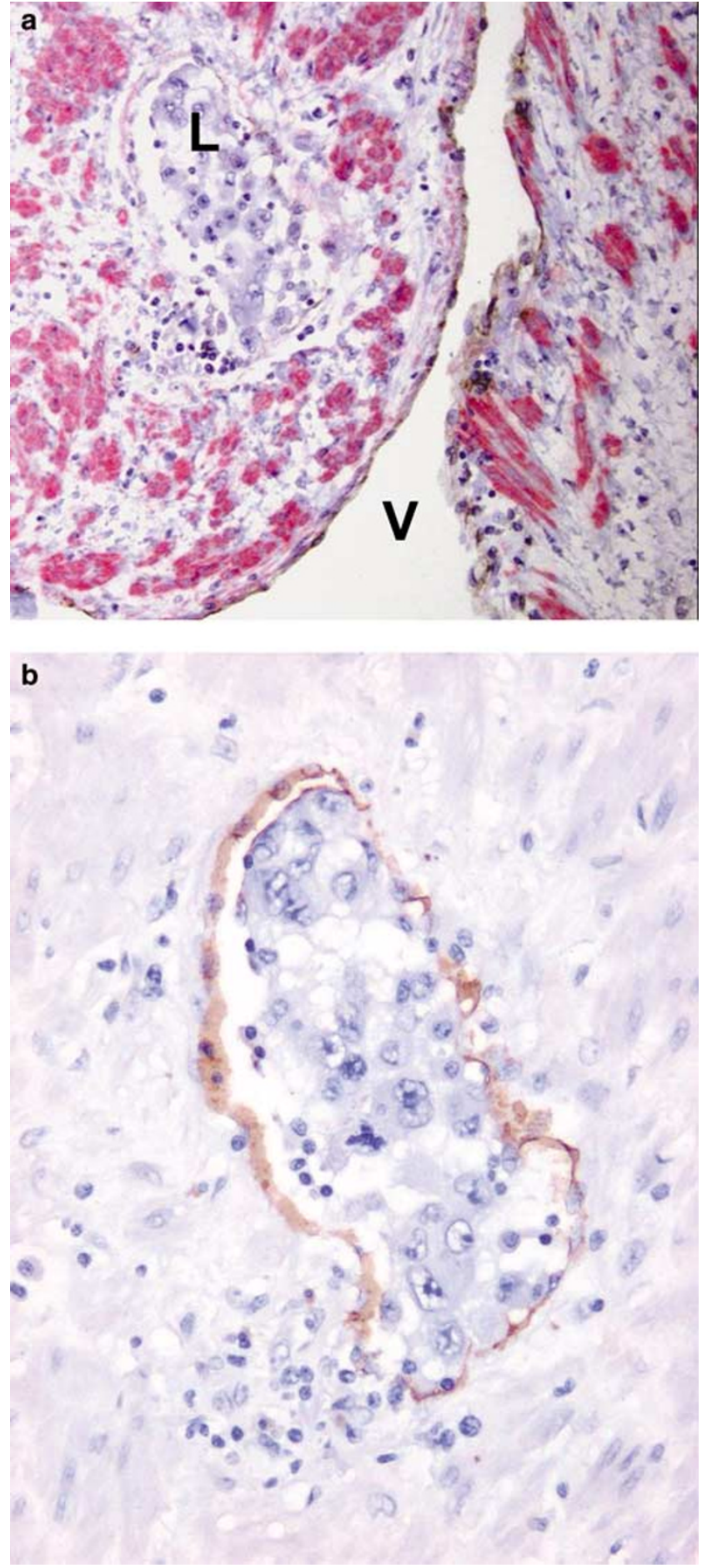

Figure 12 (a) The media of this muscular sinus vein (V) contains a tumor filled lymphatic. Immunoperoxidase stain for smooth muscle actin (red) and CD 31 (black). (b) Same nest of tumor shown in 12A. Podoplanin stain confirms intralymphatic tumor within the media of a muscular vein. Podoplanin immunoperoxidase stain.

tance. ${ }^{1,2,17,18}$ Thompson et $a{ }^{18}{ }^{18}$ in a series of 205 patients, found that 43 patients with sinus invasion had 26\% 5-year survivals, a much lower figure than previously shown for pT3 tumors. As sinus invasion

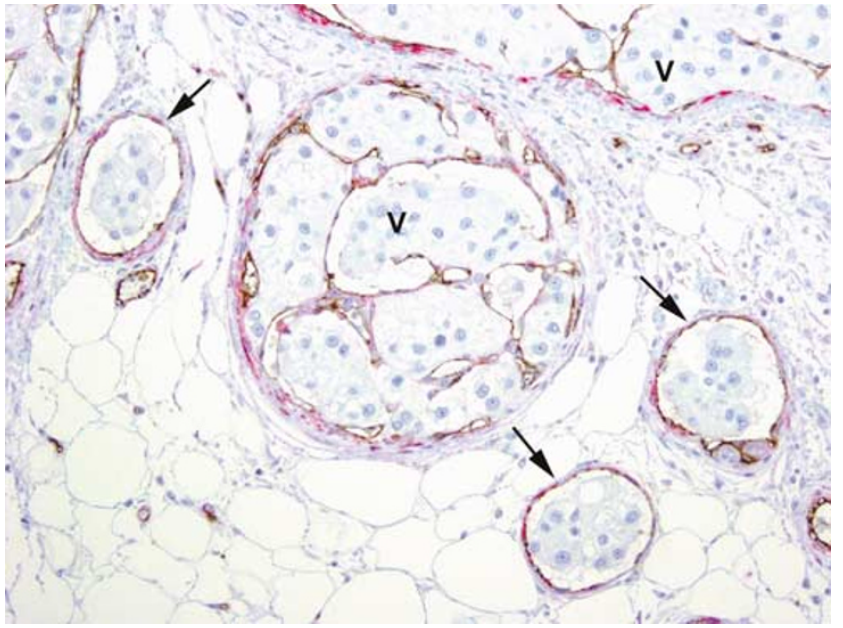

Figure 13 Intravenous tumor within larger involved veins (V) is nourished by a delicate capillary plexis. The three other involved lymphovascular structures (arrows) lacking a capillary plexis, could either be hematogenous or lymphatic in origin. Immunoperoxidase stain for smooth muscle actin (red) and CD 31 (black).

Table 1 Follow-up data: 32 cases

\begin{tabular}{|c|c|c|c|c|c|c|}
\hline & $\#$ & $\begin{array}{c}<1 \\
\text { year }\end{array}$ & $\begin{array}{c}1-2 \\
\text { year }\end{array}$ & $\begin{array}{l}2-5 \\
\text { year }\end{array}$ & $\begin{array}{c}>5 \\
\text { year }\end{array}$ & Ave follow-up \\
\hline Dead of disease & 6 & 2 & 1 & 3 & 0 & 30 months \\
\hline Metastases & 12 & 6 & 5 & 1 & 0 & 17 months \\
\hline Alive and well & 14 & 9 & 2 & 1 & 2 & 14 months \\
\hline
\end{tabular}

is usually associated with sinus vein invasion, it is likely responsible for hematogenous metastases, the most common route of tumor dissemination in CC. Renal cell carcinoma, however, also spreads by lymphatic routes; from $7-17 \%$ patients with lymph node dissections have hilar or locoregional lymph node metastases. ${ }^{3,4}$ As the major lymphatic system drains from the cortex into the renal sinus and then to regional lymph nodes, it is possible that some renal sinus structures involved by tumor may actually represent lymphatics, not veins. The development of endothelial cell markers specific for lymphatic endothelium permits investigation of this possibility. Podoplanin, a $38 \mathrm{kDa}$ membrane glycoprotein first identified on podocytes, was selected for this study because it has been shown to reliably distinguish vascular endothelium from lymphatic endothelium. ${ }^{9}$

The lymphatic organization in the kidney has been investigated in several mammals (primarily dogs, pigs, and sheep) by diverse methodologies including standard morphologic studies with light and electron microscopy, injection studies with or without dissection or digestion procedures, and microangiography. ${ }^{5-7,19-21}$ Studies of lymphatics in humans are few, most recently employing immunohistochemistry applied to renal biopsies. ${ }^{22}$ These investigations are in agreement that the major 
cortical lymphatic system consists of an anastomosing periarterial system that courses from the cortical intralobular arteries, to the corticomedullary junction, and follows the arcuate and intralobar arteries into the renal sinus. From there drainage continues to hilar lymph nodes. There are points of contention, however, such as the presence of, or quantity of, lymphatics within the cortex distant from arterial adventitia and within the renal medulla. As only limited immunohistochemical data exists regarding the normal distribution of renal lymphatics, a pilot investigation was performed as part of this study.

Control kidneys indicated that lymphatics predominately populate the periarterial sheaths beginning in the midcortex. The lymphatics enlarge and increase in number as they descend to the corticomedullary junction. Lymphatics were not observed among glomeruli or cortical tubules, or within the medulla, except when associated with zones of inflammation. Lymphatics appear to stray from a periarterial location at the corticomedullary junction. The parenchymal lymphatics were smaller than the adjacent veins at all levels in the cortex and never had a smooth muscle media. Within the renal sinus fat, lymphatics showed no apparent perivascular association; they were observed scattered throughout the fat and varied greatly in size. Lymphatics were numerous within the muscularis of the renal pelvis, and observed within the media of large muscular veins. The latter finding suggests that some sinus lymphatic tumor emboli may have a hematogenous destination and also provide an avenue for periureteral spread.

Although lymphatics were not identified within any primary tumors, clusters of small to mediumsized lymphatics were commonly identified within inflamed cortex and inflamed renal sinus both outside a pseudocapsule and intermingled with tumor cells at an invasive front. Some of these are regarded as newly formed lymphatics. As native renal lymphatics are normally restricted to the adventitia of cortical arteries, when lymphatics are identified amidst glomeruli and tubules and not in a periarterial location, they may be justifiably regarded as newly formed. Distinguishing native lymphatics from newly formed lymphatics may be more difficult in other anatomic sites where increased lymphatic density compared to normal, or lymphatic endothelial cell proliferation have been demonstrated, providing evidence for lymphangiogenesis in other neoplasms. ${ }^{14-16,23}$

The origin of newly formed lymphatics is uncertain. Embryonic lymphangiogenesis can derive either from lymphangioblasts, or from vascular endothelium by sprouting with subsequent lymphatic endothelial differentiation. ${ }^{8,11,22,23}$ As newly formed renal lymphatics arise in a heavily vascularized region such as the renal cortex and medulla where native lymphatics are not demonstrated, vascular sprouting may be responsible. The stimulus to lymphangiogenesis can be multifactorial; both tumor-related and inflammatory-related processes have been described. Tumors can produce growth factors known to induce lymphangiogenesis such as vascular endothelial growth factor C (VEGF-C). Tumor VEGF-C overexpression has been associated with lymphangiogenesis, lymphatic enlargement, increased lymphatic density, and lymph node metastases in several carcinomas, including cervix, papillary thyroid, prostate, and head and neck squamous cell carcinomas, and in melanoma. ${ }^{12-16,23}$ Lymphangiogenesis has also been associated with renal inflammatory processes unrelated to neoplasia, owing to VEGF-C production by histiocytes. ${ }^{24}$ This is likely a contributing factor in lymphangiogenesis observed in renal transplant rejection. ${ }^{22}$

In seven cases intralymphatic tumor was observed. Both peritumoral lymphatics and lymphatics distant from tumor were involved which may differ in their potential for lymphatic dissemination. Many peritumoral lymphatics may be newly formed, a conclusion based upon their cortical location and association with inflammation. If correct, their functionality is uncertain, that is, the patency of their connection to veins or lymphatics. As peritumoral lymphatics within the invasive front were irregular and often appeared incomplete, the impression is conveyed that they were caught in the path of destructive tumor growth and may be soon destined for obliteration. Assuming tumor could travel thru newly formed lymphatics, their prognostic importance could relate to hematogenous spread rather than lymphatic spread if they sprouted from the venous system. As all of these cases demonstrated extensive large vein involvement, an additive adverse prognosis of a few involved peritumoral lymphatics seems unlikely.

Tumors cells within intact native lymphatics have the potential for dissemination. Convincing native lymphatic tumor emboli was identified in four cases. Support for the contention that these represent lymphatic spread was location, since these lymphatics were separate from the primary tumor within sinus fat, and within lymphatics of the intralobular arterial adventitia, pelvic muscularis, and media of large muscular sinus veins. Lymphatic involvement of these sites implies the potential for wider dissemination, both to regional nodes and along periureteral pathways in the case of the pelvic muscularis involvement. Although lymphatic emboli was an infrequent finding in this study, four of 40 cases $(10 \%)$, this is within the reported range of hilar and loco-regional lymph node involvement of $7-17 \%$ based upon lymph node dissections.,

This study suggests that intravenous tumor can often be distinguished from intralymphatic tumor based upon size of the involved structure, or by histologic features. All grossly visible involved structures within the sinus fat believed to represent veins were shown to be veins in this study. Although medium-sized sinus lymphatics, 1-2 $\mathrm{mm}$ were identified, no involved lymphatics larger than 
$0.5 \mathrm{~mm}$ were encountered. When histological examination reveals an involved lymphovascular structure, the presence of an intraluminal capillary plexis was completely discriminatory since it was restricted to intravenous tumor. However, when tumor involves the small venules and hematogenous capillaries, a capillary plexus is absent precluding separation from lymphatics without a lymphatic endothelial cell marker.

In summary, intralymphatic tumor can be demonstrated in $10 \%$ of pT3b CC. Lymphatic involvement is not only less frequent than venous involvement, it is less extensive and the involved structures are much smaller than the involved veins and always lack a capillary plexis. The potential for lymphatic spread may not be equal among involved lymphatics. The smallest involved lymphatics, the peritumoral lymphatics, are not associated with lymph node involvement and appear destined for destruction by continued tumor growth. Conversely, intralymphatic tumor emboli within the renal sinus appear prognostically important because they correlated with extensive (20 of 42 lymph nodes) lymph node involvement. However, failure did to identify lymphatic involvement did not predict absence of lymphatic spread, since lymph nodes were involved in five of 33 cases without demonstrable lymphatic involvement, although in each case only a single node was affected.

\section{References}

1 Bonsib SM. The renal sinus is the principal invasive pathway: a prospective study of 100 renal cell carcinomas. Am J Surg Pathol 2004;28:1594-1600.

2 Bonsib SM, Gibson D, Mhoon M, et al. Renal sinus involvement in renal cell carcinomas. Am J Surg Pathol 2000;24:451-458.

3 Matsuyama H, Hirata H, Korenaga Y, et al. Clinical significance of lymph node dissection in renal cell carcinoma. Scand J Urol Nephrol 2005;39:30-35.

4 Minervini A, Lilas L, Morelli G, et al. Regional lymph node dissection in the treatment of renal cell carcinoma: is it useful in patients with no suspected adenopathy before or during surgery? BJU Int 2001;88: 169-172.

5 Albertine KH, O’Morchoe CC. Distribution and density of the canine renal cortical lymphatic system. Kidney Int 1979;16:470-480.

6 Albertine KH, O’Morchoe CC. An integrated light and electron microscopic study on the existence of intramedullary lymphatics in the dog kidney. Lymphology 1980;13:100-106.

7 O'Morchoe CC, Albertine KH. The renal cortical lymphatic system in dogs with unimpeded lymph and urine flow. Anat Rec 1980;198:427-438.
8 Al-Rawi MA, Mansel RE, Jiang WG. Molecular and cellular mechanisms of lymphangiogenesis. Eur J Surg Oncol 2005;31:117-121.

9 Evangelou E, Kyzas PA, Trikalinos TA. Comparison of the diagnostic accuracy of lymphatic endothelium markers: Bayesian approach. Mod Pathol 2005;18: 1490-1497.

10 Karkkainen MJ, Alitalo K. Lymphatic endothelial regulation, lymphoedema, and lymph node metastasis. Semin Cell Dev Biol 2002;13:9-18.

11 Oliver G, Detmar M. The rediscovery of the lymphatic system: old and new insights into the development and biological function of the lymphatic vasculature. Genes Dev 2002;16:773-783.

12 Birner P, Obermair A, Schindl M, et al. Selective immunohistochemical staining of blood and lymphatic vessels reveals independent prognostic influence of blood and lymphatic vessel invasion in early-stage cervical cancer. Clin Cancer Res 2001;7:93-97.

13 Dadras SS, Lange-Asschenfeldt B, Velasco P, et al. Tumor lymphangiogenesis predicts melanoma metastasis to sentinel lymph nodes. Mod Pathol 2005;18: 1232-1242.

14 Kyzas PA, Geleff S, Batistatou A, et al. Evidence for lymphangiogenesis and its prognostic implications in head and neck squamous cell carcinoma. J Pathol 2005;206:170-177.

15 Yasuoka H, Nakamura $\mathrm{Y}$, Zuo $\mathrm{H}$, et al. VEGF-D expression and lymph vessels play an important role for lymph node metastasis in papillary thyroid carcinoma. Mod Pathol 2005;18:1127-1133.

16 Zeng Y, Opeskin K, Horvath LG, et al. Lymphatic vessel density and lymph node metastasis in prostate cancer. Prostate 2005;65:222-230.

17 Bonsib SM. Risk and prognosis in renal neoplasms. A pathologist's prospective. Urol Clin North Am 1999;26: 643-660, viii.

18 Thompson RH, Leibovich BC, Cheville JC, et al. Is renal sinus fat invasion the same as perinephric fat invasion for pT3a renal cell carcinoma? J Urol 2005; 174:1218-1221.

19 Bell RD, Keyl MJ, Shrader FR, et al. Renal lymphatics: the internal distribution. Nephron 1968;5:454-463.

20 Clark RL, Cuttino Jr JT. Microradiographic studies of renal lymphatics. Radiology 1977;124:307-311.

21 Cuttino Jr JT, Jennette JC, Clark RL, et al. Renal medullary lymphatics: microradiographic, light, and electron microscopic studies in pigs. Lymphology 1985;18:24-30.

22 Kerjaschki D, Regele HM, Moosberger I, et al. Lymphatic neoangiogenesis in human kidney transplants is associated with immunologically active lymphocytic infiltrates. J Am Soc Nephrol 2004;15: 603-612.

23 Al-Rawi MA, Mansel RE, Jiang WG. Lymphangiogenesis and its role in cancer. Histol Histopathol 2005;20:283-298.

24 Schoppmann SF, Birner P, Stockl J, et al. Tumorassociated macrophages express lymphatic endothelial growth factors and are related to peritumoral lymphangiogenesis. Am J Pathol 2002;161:947-956. 